\title{
Stochastic Metallic-Glass Cellular Structures Exhibiting Benchmark Strength
}

\author{
Marios D. Demetriou, ${ }^{*}$ Chris Veazey, John S. Harmon, Joseph P. Schramm, and William L. Johnson \\ Keck Laboratory, California Institute of Technology, Pasadena, California 91125, USA
}

(Received 11 February 2008; revised manuscript received 17 June 2008; published 3 October 2008)

\begin{abstract}
By identifying the key characteristic "structural scales" that dictate the resistance of a porous metallic glass against buckling and fracture, stochastic highly porous metallic-glass structures are designed capable of yielding plastically and inheriting the high plastic yield strength of the amorphous metal. The strengths attainable by the present foams appear to equal or exceed those by highly engineered metal foams such as Ti-6Al-4V or ferrous-metal foams at comparable levels of porosity, placing the present metallic-glass foams among the strongest foams known to date.
\end{abstract}

The fundamentals governing the strength capabilities of foam materials have been well studied over the past three decades [1]. Depending on the mechanism accommodating foam failure, the failure stress will be determined by the relevant structural property of the parent solid. Specifically, the strength of a plastically yielding foam will be determined by the solid yield strength, the strength of a brittle foam by the solid fracture stress, and the strength of an elastically buckling foam by the solid modulus. Since fracture and buckling stresses of solids are generally lower than plastic yield strengths, brittle or elastically buckling foams tend to be weaker than plastically yielding foams. Owing to remarkably high plastic yield strengths, amorphous metals are thought to be attractive parent materials for ultrastrong foams [2]. Considerable advances in the synthesis, characterization, and testing of metallic-glass foam have been reported to date [3-14]. Low- to moderate-porosity foams are found to exhibit strengths that are roughly consistent with the high plastic yield strength of the monolithic amorphous metal [7-12]; however, higher-porosity foams ( $>80 \%$ ) fail at relatively low stresses that cannot be correlated to the strength of the parent solid $[13,14]$. In this Letter, we demonstrate that, by matching the key characteristic "structural scales" that dictate the resistance of a metallic-glass cellular structure against buckling and fracture, the metallic-glass foam can inherit the plastic yield strength of the amorphous metal up to porosities as high as $92 \%$. The achievable foam strengths reported herein appear to equal or exceed those by strong highly engineered metal foams, placing the present metallic-glass foams among the strongest foams of any kind.

Amorphous metals exhibit superb plastic yield strengths; however, upon unconfined loading they fail plastically by shear localization attaining very limited global plasticity terminated by brittle fracture. Unlike ceramics, though, most amorphous metals are capable of undergoing plastic yielding prior to failing catastrophically by fracture, an ability attributed to a toughness adequate to suppress fracture until plasticity is initiated. Fracture toughness values for amorphous metals typically range between 10 and $100 \mathrm{MPa} \mathrm{m}^{1 / 2}$, going from relatively brittle to relatively tough alloys [15]. Such modest fracture toughness values combined with high plastic yield strengths give rise to rather small plastic zone thicknesses $t_{\mathrm{cr}}$, ranging from $10^{-6}$ to $10^{-3} \mathrm{~m}$ going from brittle to tough alloys [15]. Not surprisingly, the attainable plastic deformability of a metallic-glass column in bending is found to be dramatically enhanced as the column thickness approaches $t_{\mathrm{cr}}$, an effect attributed to geometric suppression of crack nucleation following plastic yielding [16]. In connection to a cellular metallic-glass structure, the ratio of the average strut thickness to the plastic zone thickness $\bar{t} / t_{\mathrm{cr}}$ can thus be thought as a key structural scale that dictates the foam's resistance against catastrophic fracture. That is, for a metallic-glass cellular structure to be able to resist catastrophic fracture, $\bar{t}$ should be comparable to or lower than $t_{\mathrm{cr}}$. The significance of $\bar{t} / t_{\mathrm{cr}}$ as a characteristic scale of the metallic-glass cellular structure has been well emphasized in prior literature $[2,13,14]$. Nevertheless, consideration of this structural scale alone may not be sufficient to determine the overall strength capabilities of a metallic-glass foam.

In addition to unusually high plastic yield strengths, amorphous metals also exhibit rather low elastic moduli giving rise to relatively high elastic strain limits $\varepsilon_{\mathrm{el}}$. A universal $\varepsilon_{\mathrm{el}}$ of $\sim 0.02$ has been recognized for all amorphous metals [15], a limit substantially higher than most conventional crystalline metals whose limits are typically bounded well below 0.01 . Considering such high $\varepsilon_{\mathrm{el}}$, one would expect metallic-glass columns to be susceptible to elastic buckling at a relatively low slenderness ratio. The critical slenderness ratio that determines the stability of a column against elastic buckling is given by $(l / t)_{\mathrm{cr}}=$ $n \pi / \sqrt{\varepsilon_{\mathrm{el}}}$, where the index $n$ depends on the end constraints and ranges between $1 / 2$ and 2 [17]. Using $\varepsilon_{\mathrm{el}}=0.02$, one can estimate $(l / t)_{\mathrm{cr}}$ for a metallic-glass column to range between 20 and 40 . In contrast, $(l / t)_{\text {cr }}$ for an aluminum column with $\varepsilon_{\mathrm{el}}=0.005$ can be estimated to range between 40 and 80. Amorphous metals therefore due to their 
high elastic limit are more prone to buckling than conventional metals when subjected to the same loading constraints. In principle, a highly porous cellular structure consisting of struts whose average aspect ratio is comparable to or greater than $(l / t)_{\text {cr }}$ would be unstable against cooperative buckling. Using established scaling relations [1], the critical relative density above which cellular structures tend to fail cooperatively by buckling can be estimated to be $\sim\left(6 \varepsilon_{\mathrm{el}}\right)^{2}$, which for a metallic-glass structure corresponds to $\sim 0.01$ ( $\sim 99 \%$ porosity). In practice, however, a morphologically irregular cellular structure involving randomly distributed buckling-prone struts can fail by buckling percolation at a relative density considerably higher than the critical one. The extent to which morphological irregularities influence the buckling resistance of a cellular structure is well documented in prior literature [18]. Since amorphous metals are generally more prone to buckling than conventional metals, the effect of morphological irregularities on the buckling resistance of a metallic-glass foam would be more dramatic. Indeed, highly stochastic metallic-glass foams were found to fail by percolation of buckling instabilities at relative densities as high as 0.6 [8]. The morphological periodicity or "degree of order" of the metallic-glass cellular structure therefore represents another characteristic structural scale which dictates the structure's resistance against failure by random buckling.

In this Letter, we demonstrate that, by meeting the key structural scales identified above, highly porous metallicglass foams able to resist fracture and buckling and thus inherit the plastic yield strength of the parent glass can be developed. The $\mathrm{Pd}_{43} \mathrm{Ni}_{10} \mathrm{Cu}_{27} \mathrm{P}_{20}$ metallic-glass alloy is utilized here, and a foaming method based on thermoplastic expansion of entrained gas bubbles is employed [19]. In the present foaming method, foam expansion is performed at an optimum temperature and strain rate at which the liquid exhibits high ductility yet adequately low fluidity, enabling the development of cellular structures with features that meet the key "structural scales" identified above. Specifically, a high liquid ductility (strain-rate sensitivity exponent near unity) enables membrane drawing to thicknesses sufficiently lower than $t_{\mathrm{cr}}$, while a low liquid fluidity (high viscosity) inhibits bubble sedimentation and promotes sufficient cellular periodicity. By means of the present method, structurally regular metallic-glass foams with porosities ranging between $83 \%$ and $92 \%$ have been produced. An amorphous 88\%-porosity foam is shown in Fig. 1(a). Optical micrographs of a representative cross section of the cellular structure are presented in Figs. 1(b) and 1(c). Intracellular membranes having thicknesses on the order of tens of microns can be observed. Given that $t_{\mathrm{cr}}$ for the families of Pd- and Pt-based metallic glasses is reported to be on the order of hundreds of micrometers [15], membranes with $\bar{t} \ll t_{\text {cr }}$ will give rise to adequately high fracture resistance. Figures $1(\mathrm{~b})$ and 1(c)

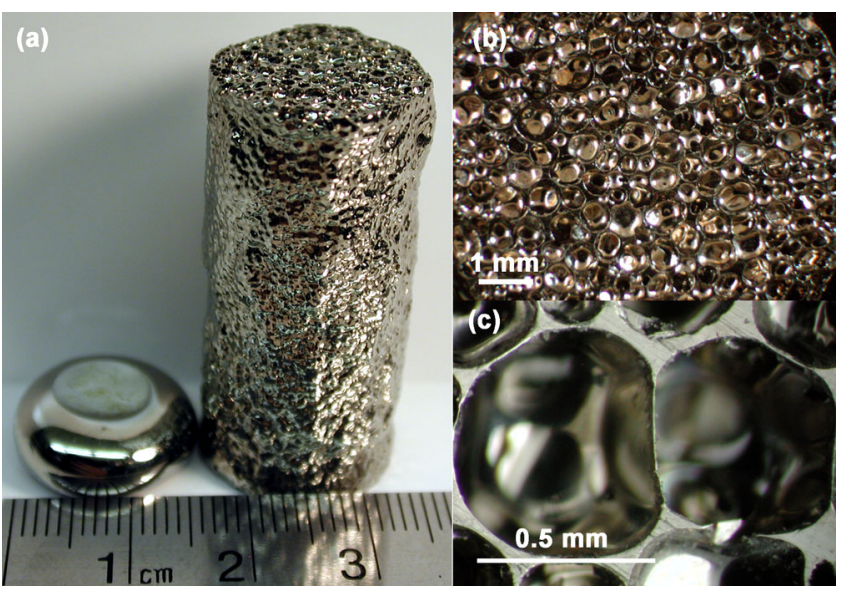

FIG. 1 (color online). (a) Image of 88\%-porosity $\mathrm{Pd}_{43} \mathrm{Ni}_{10} \mathrm{Cu}_{27} \mathrm{P}_{20}$ foam produced via the present synthesis route. A pore-free button of equivalent mass is also presented to demonstrate the tenfold increase in volume produced by foaming. (b),(c) Optical micrographs depicting a cross section of the cellular structure at different magnifications.

also qualitatively demonstrate that the distribution of cell sizes in the present structures is rather narrow: Average cell sizes of roughly $0.5 \mathrm{~mm}$ can be observed, and substantial deviations from this average size appear to be rather limited. The apparent cell-size uniformity suggests that the cellular structure exhibits good morphological periodicity, a property that we argue is associated with high resistance against random buckling.

Compressive testing of foams in the porosity range of 83\%-92\% was performed. Cylindrical specimens with polished and parallel loading surfaces having diameters of $18 \mathrm{~mm}$ and heights ranging between 25 and $30 \mathrm{~mm}$ were prepared for mechanical testing. A servo-hydraulic materials testing system with a $50-\mathrm{kN}$ load cell was utilized. Strain rates of $1 \times 10^{-4} \mathrm{~s}^{-1}$ were applied. Strains were measured using a linear variable differential transformer. The compressive stress-strain responses of $83 \%$ and $92 \%$ porosity foams are shown in Fig. 2. The stressstrain responses reveal that the foams can be loaded elastically up to a high failure stress (occurring at approximately $1 \%$ strain), at which point they collapse and continue to deform by repetitive noncatastrophic collapse events occurring at a high plateau stress. Interestingly, the yielding response of the present foams prior to collapse does not exhibit the characteristic extended stress plateau seen in other highly stochastic metallic-glass foams $[8,10,11]$, which has been associated with the percolation of random buckling instabilities [8]. Given the absence of such a characteristic stress plateau in the loading response, one can argue that the present foams effectively evade failure by random buckling. A question then arises as to which mechanism is primarily responsible for the failure of these foams: plasticity or fracture? 


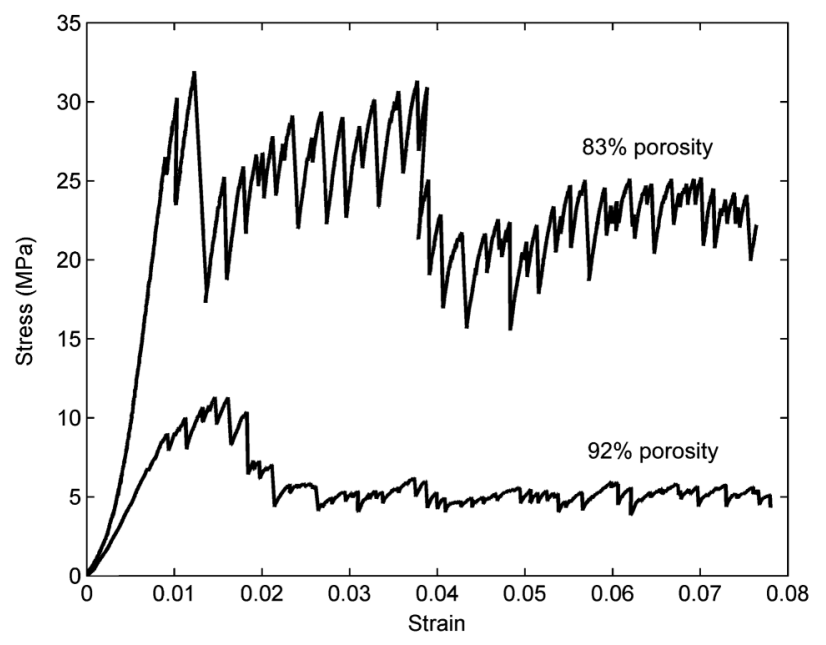

FIG. 2. Compressive stress-strain responses of $83 \%$ and $92 \%$ porosity foams.

In Fig. 3(a), the image of a collapsed foam specimen is presented. The features of a propagated collapse band are examined microscopically using scanning electron microscopy. The micrograph of Fig. 3(b) presents a cross section that reveals a propagated band of crushed cells. As shown in the micrograph of Fig. 3(c), crashed cells are associated with large crack offsets revealing that fracture is indeed one of the main mechanisms of structural failure. In the micrograph of Fig. 3(d), however, multiple shear bands are detected in the vicinity of a fracture plane revealing that considerable plasticity is realized in conjunction with frac-
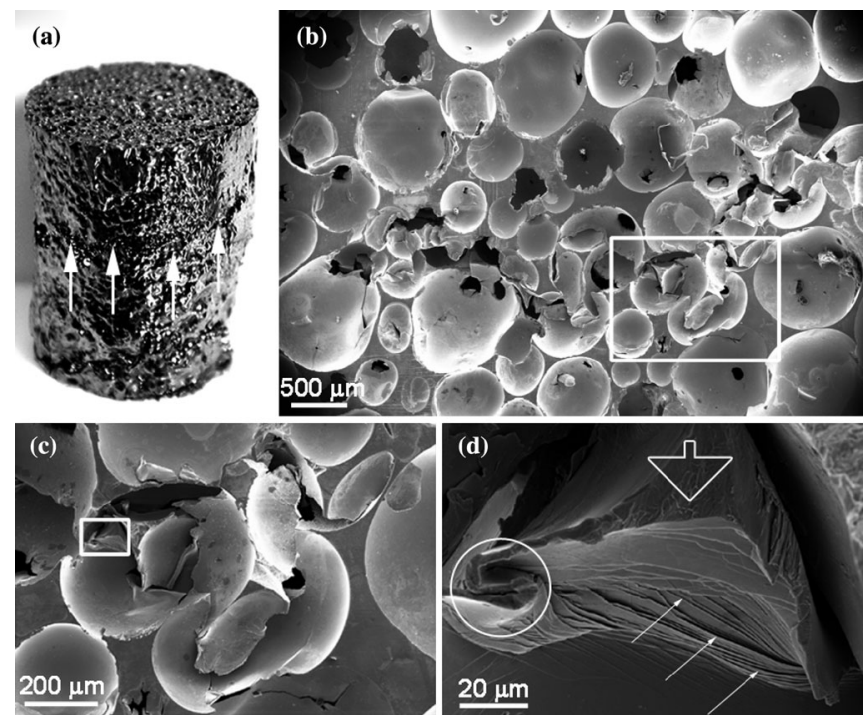

FIG. 3. (a) Image of a collapsed foam specimen (arrows indicate a macroscopically propagated collapse band). (b)(d) Scanning electron micrographs depicting features of a propagated collapse band at different magnifications. Image (d) highlights a fracture plane (thick arrow), dense shear band networks (line arrows), and a membrane that has undergone severe plastic deformation (circle). ture. The presence of such dense shear band networks suggests that failure may be initiated by plasticity despite the fact that collapse is ultimately terminated by fracture.

If failure of the present foams is indeed triggered by plasticity, the foam failure stresses should scale with the plastic yield strength of the parent amorphous metal. We thus attempt to investigate such a scaling relation. In Fig. 4, the foam failure stresses (peak stresses) normalized by the solid plastic yield strength are plotted against the foam relative densities (the yield strength and density of $\mathrm{Pd}_{43} \mathrm{Ni}_{10} \mathrm{Cu}_{27} \mathrm{P}_{20}$ glass are taken as $1630 \mathrm{MPa}$ [10] and $9.34 \mathrm{~g} / \mathrm{cc}$ [3], respectively). The power-law correlation for plastically yielding foams established by Gibson and Ashby [1] (power exponent $=1.5$; preexponential constant $=0.3$ ) is also plotted in Fig. 4. The data appear to conform well to the established correlation, indicating that the strengths of the present foams scale consistently with the plastic yield strength of the parent solid, thereby supporting that these foams are indeed plastically yieldable. Previously reported data for amorphous Zr-based foams [14], which are also plotted in Fig. 4, deviate substantially from the established correlation suggesting that plasticity is perhaps not the dominant failure mechanism for those foams.

The ability of the present foams to effectively inherit the high plastic yield strength of the parent amorphous metal results in foam strengths that are remarkably high with respect to the foam porosities. We can demonstrate here that, at this level of porosity ( $80 \%-95 \%)$, the present foams appear to be among the strongest foams of any kind. In Fig. 5, we plot the strength vs relative density data for the present foams along with the data for other foams at

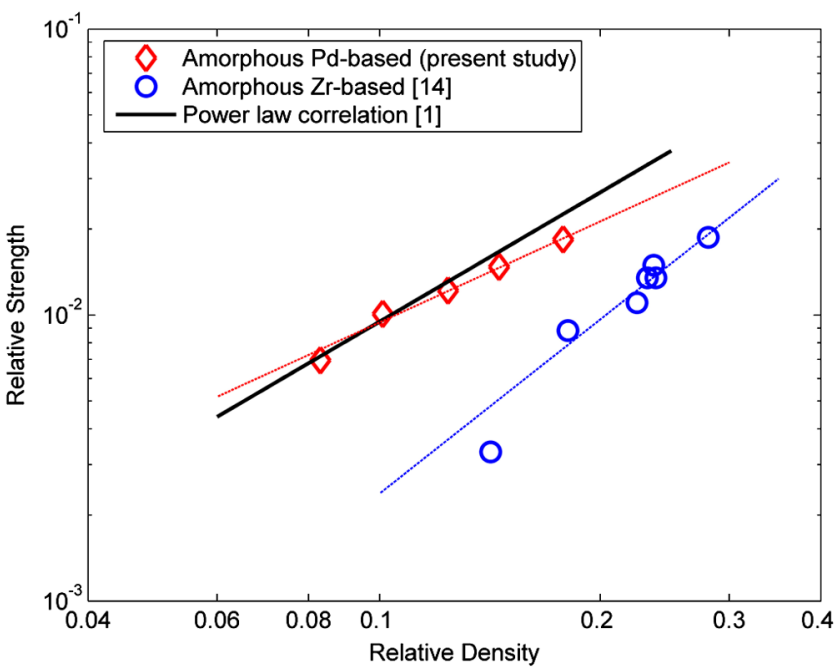

FIG. 4 (color online). Relative strength vs relative density data for the present Pd-based foams plotted together with data for previously reported Zr-based foams [14]. The dotted lines represent power-law fits to the two sets of experimental data, while the solid line is the power-law correlation for plastically yielding foams [1]. 


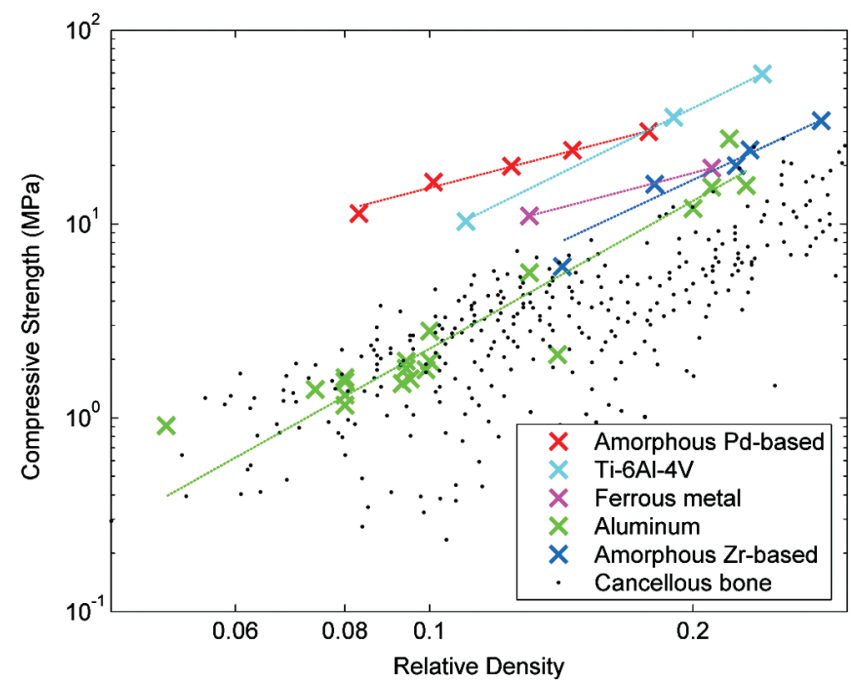

FIG. 5 (color). Strength vs relative density data for the present Pd-based foams, plotted together with data for Ti-6Al-4V foams [20], ferrous-metal foams [21], previously reported Zr-based metallic-glass foams [14], aluminum foams [22], and cancellous bone [23]. The dotted lines represent power-law fits to the sets of experimental data.

comparable levels of porosity, including Ti-6Al-4V foams produced by multiple coating techniques [20], ferrousmetal foams produced by reduction of chemically bonded ceramic foams [21], previously reported metallic-glass foams [14], conventional aluminum foams [22], and cancellous bone [23]. As seen in the plot, the present metallicglass foams exhibit strengths that surpass even those of highly engineered Ti-6Al-4V or ferrous-metal foams.

In conclusion, by matching the key characteristic structural scales that dictate the resistance of a metallic-glass cellular structure against buckling and fracture, foams capable of yielding plastically and thus inheriting the plastic yield strength of the amorphous metal up to porosity levels as high as $92 \%$ are produced. The strengths attainable by the present foams appear to equal or exceed those by strong highly engineered metal foams such as Ti-6Al$4 \mathrm{~V}$ or ferrous-metal foams, placing the present foams among the strongest foams known to date.

The authors acknowledge valuable discussions with R. D. Conner, J.C. Hanan, E. Üstündag, J. Schroers, S. Bossuyt, K. Samwer, R. Birringer, and L. A. Dunning and express their gratitude to G. Ravichandran for providing the testing apparatus and to L. Chan-Hou for the valuable assistance. This work was supported by the Office of Naval Research under Grant No. N00014-07-11115 .

\footnotetext{
*marios@caltech.edu
}

[1] L. J. Gibson and M.F. Ashby, Cellular Solids: Structure and Properties (Cambridge University Press, Cambridge, United Kingdom, 1997), 2nd ed., Chap. 5.

[2] A. H. Brothers and D.C. Dunand, Scr. Mater. 54, 513 (2006).

[3] J. Schroers, C. Veazey, and W. L. Johnson, Appl. Phys. Lett. 82, 370 (2003).

[4] J. Schroers, C. Veazey, M.D. Demetriou, and W.L. Johnson, J. Appl. Phys. 96, 7723 (2004).

[5] M. D. Demetriou, C. Veazey, J. Schroers, J. C. Hanan, and W. L. Johnson, J. Alloys Compd. 434-435, 92 (2007).

[6] M. D. Demetriou, C. Veazey, J. Schroers, J. C. Hanan, and W. L. Johnson, Mater. Sci. Eng. A 449-451, 863 (2007).

[7] M.D. Demetriou, J.P. Schramm, C. Veazey, W.L. Johnson, J.C. Hanan, and N.B. Phelps, Appl. Phys. Lett. 91, 161903 (2007).

[8] M. D. Demetriou, J. C. Hanan, C. Veazey, M. Di Michiel, N. Lenoir, E. Üstündag, and W. L. Johnson, Adv. Mater. 19, 1957 (2007).

[9] T. Wada and A. Inoue, Mater. Trans., JIM 44, 2228 (2003).

[10] T. Wada and A. Inoue, Mater. Trans., JIM 45, 2761 (2004).

[11] T. Wada and A. Inoue, Mater. Trans., JIM 46, 2777 (2005).

[12] T. Wada, M. Kinaka, and A. Inoue, J. Mater. Res. 21, 1041 (2006).

[13] A. H. Brothers and D.C. Dunand, Adv. Mater. 17, 484 (2005).

[14] A. H. Brothers and D.C. Dunand, Acta Mater. 53, 4427 (2005).

[15] M. F. Ashby and A. L. Greer, Scr. Mater. 54, 321 (2006).

[16] R. D. Conner, W. L. Johnson, N. E. Patton, and W. D. Nix, J. Appl. Phys. 94, 904 (2003).

[17] J.M. Gere and S.P. Timoshenko, Mechanics of Materials (PWS-Kent, Boston, 1975), 3rd ed., Chap. 9.

[18] A. G. Evans, J. W. Hutchinson, and M.F. Ashby, Prog. Mater. Sci. 43, 171 (1998).

[19] The alloy was prepared by first prealloying Pd $(99.9 \%$ purity), Ni (99.9\% purity), and $\mathrm{Cu}$ ( $99.99 \%$ purity) by induction melting and then alloying $\mathrm{P}$ (99.9999\% purity) by stepwise furnace heating. A button of the alloy together with $\mathrm{H}_{3} \mathrm{BO}_{3}$ powder was enclosed in a quartz tube and was inductively heated under 1-bar pressure of argon. After melting of the alloy, a bubbly two-phase mixture is generated by the decomposing acid. The tube containing the mixture was then immersed in molten tin at $420^{\circ} \mathrm{C}$ and was settled to attain thermal equilibrium for approximately $30 \mathrm{~s}$. Pressure was subsequently reduced to induce foam expansion at the controlled rate of $\sim 10^{-2} \mathrm{bar} / \mathrm{s}$. After the desired drop in pressure was attained, the foamy liquid was rapidly water quenched. Variations in porosity were achieved by varying the total drop in pressure during foaming. Porosities were assessed using the Archimedes as well as the graphical method.

[20] J. P. Li, S. H. Li, C. A. Van Blitterswijk, and K. De Groot, J. Mater. Sci., Mater. Med. 17, 179 (2006).

[21] A. Verdooren, H. M. Chan, J. L. Grenestedt, M. P. Harmer, and H. S. Caram, J. Am. Ceram. Soc. 89, 3101 (2006).

[22] T. Mukai, H. Kanahashi, T. Miyoshi, M. Mabuchi, T. G. Nieh, and K. Higashi, Scr. Mater. 40, 921 (1999).

[23] L. J. Gibson, J. Biomech. 18, 317 (1985). 